\title{
Civilisations
}

Revue internationale d'anthropologie et de sciences

humaines

$65 \mid 2016$

Figures du malentendu

\section{De la valeur heuristique du malentendu}

Retour sur un terrain au Kerala

\section{Laura Silvestri}

\section{(2) OpenEdition}

12 Journals

Édition électronique

URL : http://journals.openedition.org/civilisations/4067

DOI : $10.4000 /$ civilisations. 4067

ISSN : 2032-0442

Éditeur

Institut de sociologie de l'Université Libre de Bruxelles

\section{Édition imprimée}

Date de publication : 19 décembre 2016

Pagination : 107-126

ISBN : 2-9602017-0-3

ISSN : 0009-8140

\section{Référence électronique}

Laura Silvestri, « De la valeur heuristique du malentendu », Civilisations [En ligne], 65 | 2016, mis en ligne le 19 décembre 2019, consulté le 15 janvier 2021. URL : http://journals.openedition.org/ civilisations/4067 ; DOI : https://doi.org/10.4000/civilisations.4067

(c) Tous droits réservés 


\title{
De la valeur heuristique du malentendu
}

\author{
Retour sur un terrain au Kerala*
}

\author{
Laura SILVESTRI
}

\begin{abstract}
Résumé : En m'inspirant d'un essai de Gerald Berreman, je reprends les métaphores dramaturgiques d'Erving Goffman pour analyser les interactions sur mon terrain ethnologique au Kerala (Inde). Comme le dit Goffman, le chercheur et ses interlocuteurs sur le terrain tendent à considérer les règles de bienséance comme allant de soi, jusqu'à ce qu'une perturbation révèle les implicites de l'interaction. Avant de me rendre au Kerala j'avais été prévenue de la réticence des maîtres d'arts martiaux à transmettre leur savoir et de l'importance de gagner leur confiance. Ainsi, les premiers mois de séjour dans une école d'arts martiaux (kalarippayarru), j'ai évité de poser des questions sur les techniques et j'ai laissé le maître décider de la progression de mon apprentissage. Le maître et les autres personnels de l'école, en revanche, donnaient pour acquis que j'étais là pour apprendre rapidement l'art martial, revenir en Europe et donner des cours. Contrairement à mes attentes, ils voyaient cela comme souhaitable. Seules leurs réactions à un faux pas de ma part m'ont permis finalement de reconnaître les représentations réciproques qui avaient influencé nos interactions, et d'ouvrir ainsi les yeux sur une politique de la transmission que je n'avais pas prise en considération jusque là.
\end{abstract}

Mots-clés : interaction, malentendu, Kerala, arts martiaux, apprentissage.

\begin{abstract}
Drawing inspiration from an essay by Gerald Berreman, I use Erving Goffman's dramaturgical metaphors to analyze the interactions I experienced in my ethnological fieldwork in Kerala ( India). As Goffman points out, the researcher and the people she meets on fieldwork tend to take interaction codes for granted, until a disruption reveals some implicit assumptions. Before going to Kerala, I was forewarned about the martial arts masters' reluctance on sharing their knowledge, and about the importance of gaining their trust. Hence, on the first months of my stay in the martial arts (kalarippayarru) school I avoided asking questions, and let the master decide on my advancement. On the contrary, the master and the school's staff took for granted the fact that I was there to quickly learn the martial art, come back to Europe, and teach it. Contrary to my assumptions, they regarded that as desirable. Just their reactions to a "faux pas » on my part allowed me to recognize the mutual representations that shaped our interactions, and opened my eyes on a politics of transmission that I had not considered before.
\end{abstract}

Keywords: interaction, misunderstanding, Kerala, martial arts, learning.

* Une première version de cet article a été présentée lors du colloque Chercheur-se in situ. Immersions par corps, normes et déviances, Montpellier, 21-23 mai 2014, sous le titre : " La mise en scène d'une expérience transformatrice. Analyse goffmanienne d'un terrain au Kerala (Inde) ». Je tiens à remercier Giorgio De Martino, Anne Dubos et Gilles Tarabout, ainsi que Françoise Lauwaert, Laurent Legrain et les lecteurs anonymes pour leurs apports critiques lors de la transformation de ce papier en article. Je remercie aussi Lenaïg Orgebin pour sa relecture. Tout manque ou erreur n'engage que ma responsabilité. 
Le malentendu dont il sera question dans cet article m'est apparu comme un moment particulièrement important dans la relation d'enquête, à envisager davantage comme un outil de connaissance que comme un inconvénient (Weber, 2009). J'aimerais partager quelques réflexions que m'a inspirées mon expérience de terrain au Kerala (Inde du sud). J'y ai mené une recherche sur les conceptions du corps et de la personne dans l'art martial kalarippayarru (prononciation : kalarippayatt), m'intéressant en particulier à la manière dont ces dernières sont influencées par la transnationalisation de la discipline ${ }^{1}$, mais il ne sera pas tant question de cela, que des interactions qui se produisirent sur le terrain. Je vais montrer comment les échanges avec mes interlocuteurs ont pu paraître satisfaisants jusqu'à ce que des perturbations dans nos interactions révèlent les malentendus liés à nos attentes réciproques, permettant ainsi dans un deuxième temps de comprendre des réalités restées inaperçues.

Ma réflexion a été stimulée par l'essai de Gerald Berreman «Behind Many Masks » (1972)2, dans lequel cet auteur prend appui sur les métaphores dramaturgiques d'Erving Goffman pour reconsidérer l'enquête ethnographique qu'il avait menée dix ans auparavant dans un village himalayen. Dans cet article, Berreman adopte les concepts mis en œuvre par Goffman dans La présentation de soi (1973 [1959]), un ouvrage qui traite en particulier des interactions en face à face. Goffman commence par définir comme « représentation » les efforts d'une personne ou d'un groupe de personnes destinés à influencer l'action d'un tiers (ibid. : 23). A partir de ce noyau, il construit une série de concepts qui complètent sa métaphore dramaturgique : les acteurs qui coopèrent à une même représentation forment « une équipe » (ibid. : 81) ; les destinataires de la représentation constituent « le public » (ibid. : 23). Les acteurs peuvent s'aider de différents éléments matériels pour mener à bien leur représentation : l'apparence physique, l'aménagement des espaces exposés au regard du public, mais aussi la distinction entre les espaces accessibles au public (" la région antérieure », ibid. : 106) et ceux qui ne le sont pas (« la région postérieure » ou « coulisse », ibid. : 110). Le contrôle de l'information qui passe d'un espace à l'autre aide à « maîtriser les impressions » données au public et à prévenir toute rupture de représentation ${ }^{3}$.

Différentes raisons m'ont amenée à adopter la perspective de Goffman dans la lecture qu'en avait fait Berreman dans son article de 1972, lequel avait été présenté par Jean-Pierre Olivier de Sardan $(1995$; 2000) comme l'une des premières tentatives systématiques de revenir sur les conditions de réalisation du travail anthropologique. Berreman soumet en effet les concepts de Goffman à l'épreuve d'un terrain qui aurait été qualifié autrefois de « terrain ethnologique classique ». De plus, son lieu d'enquête se situe comme le mien dans le sous-continent indien. Enfin, il étend de manière

1 J'ai mené cette enquête dans le cadre de ma thèse de doctorat en anthropologie sociale et ethnologie, Scorrere come un fiume. Pratiche e teorie del corpo in movimento nel kalarippayarrụ, arte marziale del Kerala (Fluide comme de l'eau. Pratiques et théories du corps en mouvement dans le kalarippayarrụ, art martial du Kerala), Université de Turin et EHESS-Paris, 2013.

2 L'essai est paru la première fois en 1962 comme un petit livre autonome. Il a été republié comme introduction méthodologique à la monographie réalisée par Berreman dix ans plus tard, en 1972, portant sur le même village. Les citations dans le présent article se réfèrent à cette deuxième édition.

3 Les concepts-clés en anglais : performance (1959 : 15-16) team (ibid. : 79), audience (ibid. : 15-16), front region (ibid. : 107), back region (ibid. : 112), impression management (ibid. : 208). 
significative les notions de régions antérieure et postérieure : alors que pour Goffman il s'agit surtout d'espaces physiques délimités par des barrières matérielles ${ }^{4}$, le texte de Berreman autorise le glissement de ces concepts vers des espaces métaphoriques.

Selon Berreman, la plupart du temps l'ethnographe et les personnes avec lesquelles il coréalise son enquête remplissent les rôles d'acteur et de public tels qu'ils ont été définis dans Goffman (1973 : 23). Chaque partie a des intérêts qu'elle défend et promeut à travers la maîtrise des impressions qu'elle donne à l'autre. L'impression que l'ethnologue donne de lui-même déterminera les relations et la qualité de la compréhension auxquelles il aura accès et, par conséquent, la réussite de son travail. Berreman a vu son positionnement dans le village de l'enquête changer totalement à la suite d'un accident de parcours. Le jeune brahmane des plaines qui l'assistait dans ses recherches est tombé malade, rendant nécessaire la recherche d'un substitut. Celuici était un musulman entre deux âges. Les interactions entre les différents acteurs et les informations récoltées par l'ethnographe changèrent de manière significative selon qu'il était accompagné par l'un ou par l'autre assistant, ce qui l'amena à se former deux visions différentes du même village. Bien que plusieurs mois se soient passés depuis le début de l'enquête, ces nouvelles informations donnèrent à Berreman une image du village et des tensions entre les hautes et les basses castes totalement différente de celle qu'il s'était formée jusque là. Dans son article, on peut voir que les termes de régions antérieure et postérieure ne désignent pas uniquement les lieux physiques où il rencontrait les villageois, mais aussi les échanges qu'avaient ces derniers entre eux en sa présence et en son absence ; en d'autres termes, il ne s'agissait pas seulement de la représentation que les villageois mettaient en œuvre en présence de l'ethnologue, mais aussi de celles auxquelles ils recouraient entre eux et qu'ils protégeaient attentivement de toute perturbation extérieure. La sauvegarde des relations au sein du village est donc apparue comme l'enjeu majeur de la manière dont la communauté conduisait ses relations avec l'ethnologue.

La reprise de Goffman par Berreman offre plusieurs éléments de réflexion applicables à mon terrain kéralais, bien que les prémisses soient très différentes. A son arrivée dans le village, Berreman a eu de la peine à faire accepter sa présence avant même de pouvoir se demander comment obtenir la confiance de ses futurs informateurs : «L'ethnographe arrive parmi ses sujets comme un intrus, généralement inconnu, inattendu et, souvent, non souhaité $\rangle^{5}$. Au Kerala, la situation de départ semble se situer à l'opposé de celle qu'a rencontrée Berreman dans la région himalayenne. Dès sa formation, cet Etat indien a promu son patrimoine culturel comme ressource identitaire, puis touristique. Les étrangers sont normalement bien accueillis et leur présence est considérée comme une confirmation de l'intérêt du patrimoine local (voir Tarabout, 2006). Dans ce contexte, le kalarippayarru figure comme l'un des multiples emblèmes culturels du Kerala et l'un de ceux-ci qui connaît la plus grande reconnaissance internationale.

4 Bien qu'il considère que c'est le comportement adopté en un lieu, plus que sa situation spatiale, qui en fait une coulisse : «En adoptant le style de la coulisse, les gens peuvent transformer n'importe quelle région en coulisse » (Goffman, $1973: 125)$.

5 « The ethnographer comes to his subjects as an unknown, generally unexpected, and often unwanted intruder » (Berreman, 1972 : xxxiii). Les traductions de l'anglais sont miennes, sauf pour les textes de Goffman (1973; 1991). 
Malgré ces prémisses différentes, la perspective adoptée me permettra d'analyser la manière dont les malentendus et les faux-pas qui ponctuent les interactions de l'ethnologue sur le terrain peuvent contribuer à révéler des enjeux locaux. Dans cet article, par « représentation » il faudra entendre les efforts déployés par chaque partie pour poursuivre ses propres intérêts (réaliser une recherche, trouver des ambassadeurs pour une école d'arts martiaux), tout en cherchant à répondre aux attentes supposées de l'autre. Les régions antérieure et postérieure ne seront pas seulement des espaces matériels, mais également, comme indiqué plus haut, des espaces métaphoriques : toutes les modalités de réalisation des interactions (choix du moment, sélection du public, organisation du quotidien) qui peuvent influencer la manière dont des informations sont révélées ou retenues.

Dans les paragraphes qui suivent, je présenterai d'abord le kalarippayarru et son développement dans le contexte régional, puis le terrain de l'enquête. Enfin je porterai mon attention sur la tension entre les modèles et la réalité de l'enseignement et je montrerai comment celle-ci, spécialement à l'heure où cette discipline suscite un intérêt croissant à l'international, a engendré des malentendus pendant mon travail de terrain.

\section{Le ka!arippayarrụ et la patrimonisalisation des arts kéralais}

Ce qui est aujourd'hui connu sous le nom de kalarippayarru est un ensemble de techniques d'entraînement au combat codifié dans les années 1930, en recueillant et en systématisant ce qui restait des techniques enseignées dans la vieille institution guerrière des kalari. Dans la période du plus grand développement de cette institution, au $16^{\mathrm{e}}$ siècle, les kaḷari, un par village, étaient à la fois des centres de formation des guerriers, des écoles et des lieux de culte (Tarabout, 1986 : 413-427 ; 1991 : 95-98). A l'époque, la côte du Malabar (l'actuel Kerala) était caractérisée par la présence de plusieurs royaumes ; les grandes entités politiques n'ayant commencé à se former qu'au $18^{\mathrm{e}}$ siècle. Cela, en plus de l'arrivée des Européens, déterminera la démobilisation progressive du système des kalari, qui se produira au début du $19^{\text {e }}$ siècle (Bayly, 1989 : 460). Les années 1930, parallèlement au mouvement pour l'indépendance de l'Inde, voient la récupération d'une partie des techniques enseignées dans les kalari et leur réinvention partielle ${ }^{6}$, mais dans un but tout autre que belliqueux. Le kalarippayarru devient un emblème culturel du Kerala. Il en incarne l'esprit guerrier dans des démonstrations spectaculaires, qui montrent aussi l'efficacité de cette méthode d'éducation physique. Une association de pratiquants, la Kerala Kalarippayatt Association, est créée en 1958 sous la tutelle du Kerala Sports Council (Zarrilli, 1998 : 57).

A partir des années 1970, la discipline commence à devenir populaire en dehors du Kerala, en suscitant de plus en plus d'intérêt auprès des artistes, essentiellement danseurs et comédiens. Le premier à écrire sur le sujet, dans le domaine des performance studies, est Phillip Zarrilli qui se rend au Kerala pour apprendre l'art dramatique du kathakali,

6 Les acteurs les plus célébrés de la codification du kalarippayarru sont C.V. Narayanan Nayar (19051944) avec son maître Kottakkal Kanaran Gurukkal (1850-1935) et Chirakkal T. Sreedharan Nair (1909-1984). 
et décide finalement de consacrer sa thèse de doctorat ${ }^{7}$ au kalarippayarru. Acteur et metteur en scène, professeur à l'Université d'Exeter, Phillip Zarrilli est aussi l'auteur de la première monographie académique consacrée à cette discipline (1998) et il a mis au point une méthode de formation de l'acteur qui intègre le kalarippayarru, le yoga et le taijiquan (Zarrilli, 2008). A l'heure actuelle le kalarippayarru est utilisé comme une méthode complémentaire à l'entraînement habituel par certains artistes, qu'ils soient indiens ou non. Un nombre croissant d'étrangers (comédiens, danseurs, pratiquants d'arts martiaux et de $\operatorname{yog} a$ ) se rend au Kerala pour apprendre le kalarippayarru lors de séjours plus ou moins longs.

La valorisation du patrimoine du Kerala et l'attrait qu'il exerce sur la scène internationale ne concernent pas que le kalarippayarru. Au contraire, les gouvernements d'orientation marxiste qui se sont succédés au Kerala dès la création de l'Etat en 1956, ont tenu à valoriser le patrimoine immatériel et ont donné vie à un mouvement très dynamique de folklore studies (Guillebaud, 2007). Des interventions ont été mises en œuvre pour protéger et encourager de nombreux arts locaux, et non seulement les formes dites classiques. Dans ce cadre, plusieurs rituels spectaculaires ont également été valorisés comme folk arts et considérés comme faisant partie du patrimoine culturel et artistique de l'Etat, plutôt que relevant du religieux au sens strict (Ashley, 1993 ; Tarabout, 2003). La possibilité de se voir reconnaître en tant qu'artistes engendre des enjeux puissants et non exempts de conflits entre spécialistes des savoirs répertoriés dans la catégorie du folklore. Ces derniers peuvent y trouver des sources de prestige, ou du moins les mobiliser pour essayer d'améliorer leurs conditions de vie (Tarabout, 1997, 2003, 2006 ; Guillebaud, 2011).

Plusieurs intellectuels et praticiens kéralais sont donc actifs dans l'étude et la promotion du patrimoine exceptionnellement riche de cet Etat. Certains accueillent volontiers les artistes et chercheurs étrangers qui viennent chez eux pour mener des enquêtes ou pour apprendre l'une des ses nombreuses formes. Non seulement l'attention et l'intérêt de ces visiteurs sont appréciés, mais certaines écoles les reçoivent et leur permettent d'être admis derrière les coulisses - dans les deux sens, littéral et métaphorique - jusqu'à devenir partie prenante des représentations données par leurs équipes. Un petit nombre de maîtres de kalarippayarru $u$ a commencé aussi à s'organiser pour recevoir des disciples étrangers en prévoyant des parcours de formation et, en quelques cas, des facilités matérielles comme le logement et les repas. Des écoles ont obtenu la reconnaissance officielle du gouvernement indien. Elles peuvent ainsi procurer des visas étudiants aux étrangers qui souhaitent effectuer des séjours d'apprentissage de longue durée. Certaines délivrent des certificats qui n'ont pas de valeur officielle auprès des institutions indiennes ou étrangères, mais qui témoignent de la validation du parcours du pratiquant par un maître et de sa légitimité à transmettre la discipline.

Le recours aux métaphores goffmaniennes relatives à la représentation semble d'autant plus pertinent dans ce contexte, où plusieurs niveaux de mise en scène sont emboîtés : le kalarippayarru est codifié à l'origine pour représenter l'esprit guerrier kéralais, l'ensemble du patrimoine étant lui-même mis en scène à titre général ; quelques étrangers sont admis à participer à la réalisation de la représentation; le personnel

7 Zarrili, P., Kalarippayattu and the Performing Artist East and West. Thèse de doctorat non publiée, Université du Minnesota, 1978. 
d'une école se met en scène devant le public des disciples, qu'il soient indiens ou non. Le développement de l'hospitalité envers les étrangers pourrait être interprété comme une extension marquée de la région antérieure, résultat d'une maîtrise croissante de la ligne de démarcation entre celle-ci et la coulisse. Toutefois, comme je l'ai évoqué et comme nous allons le voir plus en détail, dans ce cas les deux régions ne correspondent pas forcément à des espaces matériellement identifiables.

Un autre point à prendre en considération est le suivant : si, comme le remarque Berreman (1972 : xxxv), pour chaque acteur ou pour chaque équipe l'enjeu est de maîtriser la définition que le public donnera de la situation d'interaction, le début d'une relation d'enquête sur le terrain est caractérisé par l'absence de repères communs aux deux parties qui jouent l'une pour l'autre les rôles d'acteur et de public. Ainsi, initialement chaque partie ne peut que projeter des intentions sur les comportements de l'autre et lui attribuer des attentes et des interprétations à partir desquelles elle construira ses propres comportements. Le malentendu guette alors constamment la relation ethnographique. Pour Florence Weber, il est une composante inévitable et nécessaire du travail de l'ethnologue :

Anachronismes et ethnocentrismes, à condition d'être explicités parl'observateur, ne sont pas seulement un obstacle à surmonter ou un piège à éviter : ils permettent de s'étonner, ils sont véritablement des ressorts d'intelligibilité. (Weber, 2009 : 278-279)

Pour que le malentendu puisse être explicité, cependant, il faut qu'il se révèle à un certain moment. Normalement, c'est un faux pas, une perturbation dans une représentation apparemment bien huilée qui fait ressortir les différentes interprétations que les parties en cause donnent de la situation. Goffman parle de normes de bienséance considérées « comme allant de soi, jusqu'au moment où un accident, une crise, ou une circonstance particulière se produit » $(1973: 107)^{8}$. Suite à ses analyses, le faux pas deviendra plus tard un outil expérimental de l'ethnométhodologie, justement dans le but de révéler les attentes réciproques implicites dans toute interaction sociale. Le faux pas sciemment utilisé « jette un grain de sable dans les rouages bien rodés de l'interaction » (Le Breton, 2008 : 154).

Je vais donc reprendre quelques aspects des interactions qui se sont produites sur le terrain pour voir comment la découverte des attentes réciproques aide à la compréhension de réalités qui seraient autrement restées implicites. Suivant l'invitation à la mesure lancée par Jean-Pierre Olivier de Sardan (2000 : 439), l'explicitation de la relation d'enquête sert ici au dévoilement du processus de co-construction de l'ethnographie d'une réalité locale qui ne se serait pas révélée sans un faux pas dû aux prénotions de l'ethnologue et de ses interlocuteurs.

8 Ailleurs, abordant les erreurs de cadrage non fabriquées volontairement, Goffman dit : « Remarquons que l'erreur qui explique l'auto-illusion porte en général non pas sur l'action mais sur la perception, l'action erronée étant en effet rapidement signalée et corrigée par l'entourage » (1991: 121). En absence d'une action décalée par rapport au contexte, l'auto-illusion - ou bien le malentendu - est destinée à perdurer. 


\section{L'enquête}

La partie principale de l'enquête s'est déroulée pendant huit mois et demi entre 2009 et 2010, dans une école de kalarippayarru qui vise une clientèle internationale. J'ai été mise en contact avec le maître par une disciple italienne, enseignante de yoga, qui m'a proposé de lui parler par téléphone de mon projet. Être présentée facilite les relations et, même si cela peut ne pas être un but, présenter quelqu'un peut aussi constituer une démonstration de loyauté envers le maître. Celui-ci a consenti à ma demande et a rédigé la lettre d'invitation dont j'avais besoin pour obtenir le visa d'étudiante qui me permettrait de réaliser ma recherche en participant à la vie quotidienne de l'école. Me comporter comme une apprentie parmi d'autres était un choix méthodologique, mais cela m'a permis aussi d'obtenir un visa de longue durée. Les quelques écoles qui permettent d'obtenir un visa d'étudiant sont les mieux organisées pour recevoir des personnes venant de l'étranger et sont normalement tenues par un maître qui connaît l'anglais. Cela me situait dès le départ au cœur du processus de transnationalisation de la discipline que je souhaitais étudier.

L'école en question se trouve au nord du Kerala dans la région appelée Nord Malabar, berceau des variétés de pratique dites « du nord» (vațakkan) qui constituent le noyau de l'actuel kalarippayarru. Elle a été fondée dans les années 1970 par un maître instruit dans l'une des prestigieuses écoles privées qui avaient adopté l'anglais comme principale langue d'enseignement. Désireux de revitaliser l'art martial local, il s'était rapproché de plusieurs maîtres pour apprendre un maximum de techniques. Alors que cet art était sa passion, il tirait son revenu principal d'un magasin de matériaux de construction. Les premiers disciples étrangers ont commencé à arriver dans les années 1980 et les contacts développés lui ont valu des invitations en Europe. Dans les années 2000, grâce au bouche-à-oreille, le nombre des visiteurs internationaux, hommes et femmes ${ }^{10}$, s'est considérablement accru. Les recevoir pour organiser l'apprentissage du kalarippayarru et administrer les soins liés à la tradition des kalari sont devenus ses activités principales. L'école assure non seulement les cours, mais aussi le logement, la nourriture - dont l'achat est personnellement suivi par le maître - la cuisine, le ménage, la lessive et une première consultation chez un médecin ayurvédique de la ville voisine pour les nouveaux arrivants. Autour d'un noyau de personnel stable (les trois fils du maître et ses trois disciples les plus anciens), l'école emploie de manière

9 J'aurais considéré incorrect de ne pas révéler que je préparais une recherche, ce qui a aussi probablement facilité l'obtention de la lettre d'invitation. Le maître, le personnel de l'école et mes condisciples étaient donc au courant de mon projet.

10 Le milieu kéralais du kalarippayarru est essentiellement masculin bien que les discours présentent cette discipline comme transcendant toute distinction de caste, de religion et de genre. Les Vațakkan Pātțukal ou Chants $d u$ Nord, ballades populaires datées des $16^{\mathrm{e}}-17^{\mathrm{e}}$ siècles et constituant une source importante d'informations sur la société de l'époque (Kurup et Vijayakumar, 1997 ; Mathew 1979), mentionnent le geste de quelques femmes guerrières. Cela conforte l'idée du kalarippayarru comme pratique égalitaire. Dans les faits, plusieurs filles s'entraînent dans les kalari, mais la plupart abandonnent à l'adolescence ou au moment du mariage. Les femmes qui atteignent le titre de gurukkal (maître) sont des exceptions. La situation est bien différente dans le milieu international, où les femmes pratiquant le kalarippayarr ụ sont aussi nombreuses que les hommes. Plusieurs donnent des cours dans leur pays d'origine et parmi les premiers Occidentaux à avoir reçu le titre de gurukkal, en dehors de Phillip Zarrilli, on trouve la française Cécile Gordon. 
flexible plusieurs personnes selon les variations d'affluence au cours de l'année. Il peut alors y avoir un chauffeur, un ou deux factotums, d'une à trois cuisinières ou cuisiniers, un ou deux enseignants supplémentaires, un masseur qui intervient ponctuellement dans les périodes de pointe. Quand les chambres de la maison principale sont toutes occupées, des logements complémentaires sont loués dans le village. La salle à manger, autour de laquelle sont disposées la cuisine, une salle de massage et les chambres à coucher, constitue le centre de la maison-mère ${ }^{11}$. Tout le monde a accès à l'ensemble des pièces communes mais normalement la cuisine est le royaume des employés, où les visiteurs n'entrent qu'occasionnellement pour demander de menus services ou pour aider à débarrasser la table. Cette situation présente des similitudes avec celle que décrit Goffmann dans cet exemple où le personnel d'un hôtel est libre de contredire la représentation donnée aux pensionnaires, une fois franchie la porte de la cuisine (1973 : 113-116). Dans notre cas, un seuil sépare effectivement une pièce assimilable à la région antérieure, d'une autre assimilable à la coulisse. Un élément rend toutefois la situation plus complexe : si les pensionnaires ne sont pas formellement exclus de l'accès à la cuisine, la séparation spatiale est accentuée par la séparation linguistique. Alors que dans la salle à manger résonne surtout l'anglais, la cuisine est le règne du malayalam, la langue parlée dans l'Etat du Kerala. Cette division, par ailleurs, peut bien se prolonger au-delà du seuil de la cuisine. La langue locale constitue donc une cloison invisible qui permet au maître et aux employés de se sentir dans une coulisse même en présence des pensionnaires ${ }^{12}$. Ces derniers peuvent aussi se retirer derrière une cloison linguistique si leur langue maternelle est différente de l'anglais, généralement compris par les Malayali.

La manière dont le temps quotidien est scandé contribue d'ailleurs à renforcer ces cloisons invisibles. Une journée type est caractérisée par deux séances de pratique dans le kalari : l'une, qui dure environ une heure et demie ou deux heures, a lieu le matin avant le petit-déjeuner ; l'autre, qui dure environ une heure, en fin d'après-midi. Dans plusieurs kalari la séance du matin commence avant le lever du soleil. Dans le cas en question, les instructeurs vont chercher les pensionnaires en camionnette quelques minutes après le lever du soleil pour les emmener dans l'un des deux kalari de l'école ${ }^{13}$, qui se trouvent à quinze, vingt minutes de voiture des logements. Les disciples locaux ont une seule séance qui commence à huit heures dans un kalari différent, ou, dans les périodes de plus grande affluence, avec un enseignant qui s'occupe de leur groupe. Pendant mon séjour, ce groupe était constitué de dix ou douze enfants, âgés de huit à seize ans. La fréquence de deux séances par jour est attestée par les récits les plus

11 J'ai séjourné dans cette maison durant trois mois, pour ensuite me déplacer chez une famille kéralaise qui mettait des chambres à la disposition de l'école.

12 Pendant mon séjour, il n'y a jamais eu le cas de disciples venus d'autres Etats de l'Inde. Il y avait en revanche un disciple originaire du Bengale et résidant aux Etats-Unis, dont les élèves américains viennent régulièrement dans cette école. Une femme venue du Gujarat uniquement pour les traitements thérapeutiques a séjourné dans la maison-mère.

13 Le kalari étant le lieu consacré à l'entrainement, dans la plupart des cas ce mot peut être considéré comme synonyme d'école. Lorsque j'utilise ce dernier terme c'est pour lui donner, comme le fait le maître quand il utilise le terme school, un sens plus large et désigner l'ensemble de l'organisation qui entoure le kalari. 
anciens sur les guerriers du Malabar ${ }^{14}$. Toutefois, alors que traditionnellement on ne s'entraînait que pendant la mousson ${ }^{15}$ - tradition encore respectée dans beaucoup de kaḷari, qui ferment lors des mois les plus chauds - la présence des étrangers entraîne l'ouverture de l'école tout le long de l'année. Cela permet aux visiteurs de profiter de leurs périodes de disponibilité ou de leurs congés pour commencer ou poursuivre leur apprentissage. Les cours peuvent être assurés par le maître ou par un ou plusieurs de ses assistants, normalement ses fils et ses disciples les plus anciens, et ils se déroulent selon les mêmes phases que dans les autres kalari que j'ai pu visiter, qu'ils accueillent ou non des disciples étrangers. Comme dans d'autres kalari également, selon les moments de l'année il peut y avoir un plus ou moins grand nombre d'apprentis et donc un ratio différent entre enseignants et disciples, même si l'école où j'ai fait mon apprentissage comprend sans doute un plus grand nombre d'instructeurs. Le répertoire des techniques enseignées est aussi semblable à celui de bon nombre d'autres kalari, avec en plus quelques exercices moins connus.

Trois moments de convivialité ponctuent chaque journée de l'école : le petitdéjeuner, le déjeuner et le dîner. Autour de la table se réunissent des personnes de plusieurs nationalités, qui se trouvent à vivre ensemble pour quelques jours, semaines ou mois. Le maître déjeune fréquemment avec ses hôtes, en racontant des anecdotes et en répondant avec modération aux questions posées sur la pratique. Il ne juge pas approprié de satisfaire toute la curiosité des apprentis, estimant que tout enseignement doit être transmis au bon moment. Le reste du temps est consacré essentiellement au repos ou à quelques courses dans la ville. Ponctuellement, le maître et ses collaborateurs organisent quelques visites de lieux remarquables (sanctuaires, jardin zoologique, etc.). Ces derniers aspects du quotidien de l'école sont spécifiques au type d'entreprise que le maître et son équipe ont créée. Les disciples kéralais, pour leur part, ne se rendent au kalari que pour les cours. Martin Welton (2011) remarque que même dans une école qui ne propose ni repas ni logement, le quotidien des disciples étrangers est caractérisé par le fait de ne pas être soumis aux mêmes contraintes que les disciples locaux. Les disciples internationaux rencontrés par Welton en un célèbre kalari de la capitale sont engagés dans des apprentissages artistiques ou des travaux académiques. La pratique dans le kalari constitue le but principal de leur présence sur place, alors que les disciples locaux doivent concilier leurs séances avec leurs responsabilités quotidiennes d'étudiants, de travailleurs et/ou de parents.

Dans notre cas, le temps des visiteurs est bien organisé par l'école et peu d'espace est laissé à d'éventuelles initiatives de leur part. Quand elles ont lieu, celles-ci suscitent une certaine inquiétude. Cela tient d'une part à l'hospitalité pratiquée en ces lieux, qui prescrit d'accompagner les visiteurs en permanence, notamment pour les protéger des difficultés auxquelles pourrait les exposer leur méconnaissance du contexte local, et d'autre part, au fait que les étrangers représentent à la fois une chance et un risque pour les habitants. Leur présence peut conférer du prestige à qui les fréquente, elle peut parfois apporter des revenus significatifs, ou elle peut à tout le moins susciter de la curiosité et représenter une distraction, mais elle peut aussi les amener à enfreindre

14 Par exemple le témoignage de Duarte Barbosa (1946:139-140), qui remonte au début du $16^{\mathrm{e}}$ siècle. 
indirectement plusieurs interdits ${ }^{16}$; la conduite des étrangers étant facilement perçue comme immorale. Le maître a tout intérêt à ce que les étrangers ne circulent pas seuls pour éviter que certains de leurs comportements ne portent préjudice au renom de l'école et il cherche à satisfaire toutes les exigences de ses visiteurs sans qu'ils aient besoin d'agir de manière autonome. Pour procéder de la sorte, il s'appuie sur une expérience solide. En effet, il reçoit des étrangers depuis longtemps et il a été invité à donner des démonstrations avec ses enfants et ses disciples en plusieurs pays, dont l'Italie, l'Allemagne, l'Estonie, les Philippines et le Japon ${ }^{17}$.

La compétence des habitants, tant sur le plan linguistique que dans leur capacité à prévenir les exigences des visiteurs, peut produire l'illusion que la distance culturelle n'est pas si difficile à surmonter. Pour poursuivre avec la métaphore de la représentation, le contour des rôles respectifs est attribué aux uns par les autres sur la base d'expériences précédentes. Tout fonctionne avec fluidité tant que l'une des parties ne sort pas du rôle attendu. En revanche, les écarts par rapport aux conduites et aux répliques prévues peuvent surprendre mais aussi déranger. Chaque déviation équivaut à une incursion, voulue ou non, dans une coulisse. La perturbation ouvre alors une brèche et révèle les malentendus qui peuvent se nicher dans les implicites de la représentation. Dans mon cas, la raison de ma présence (une recherche) faisait que mes intérêts et mes comportements ne correspondaient pas à mon rôle officiel d'apprentie. La relation d'apprentissage a été perturbée de manière révélatrice. Dans les paragraphes qui suivent je vais d'abord présenter quelques aspects courants de cette relation, pour ensuite revenir sur la manière dont elle s'est déclinée lors de mon enquête.

\section{Modèles et réalités de la transmission}

Selon les pratiquants avec qui j'ai pu discuter et selon les manuels de kalarippayarru écrits par des Malayali ${ }^{18}$, l'apprentissage débute idéalement à l'âge de sept ans ${ }^{19}$ et se poursuit pendant douze ans environ. Bien qu'en réalité l'invitation à la pratique soit étendue à tout le monde indépendamment de l'âge et que cette temporalité idéale ait

16 Pour ne citer qu'un exemple des plus courants, la manière de s'habiller des étrangers est souvent perçue comme inconvenante tant moralement qu'esthétiquement.

17 Un chercheur hollandais a d'ailleurs été son disciple et a rédigé un manuel pratique (Luijendijk, 2005) et une thèse de doctorat en histoire des religions (Luijendijk, 2008) sur le kalarippayarru, tous deux centrés sur cette école. Les travaux de Luijendijk se basent sur trois séjours entre 1996 et 2003. Ils ont constitué une source de comparaison incontournable qui m'a permis de comprendre l'évolution de l'école et la perspective du maître sur sa discipline au cours des années précédant ma recherche. Ils en restituent un portrait très différent de ce que j'ai pu constater en 2009-2010. En plus, Phillip Zarrilli, tout en étant principalement disciple d'une autre école, a réalisé ici une partie de sa recherche et est bien connu par le maître. Enfin, juste après mon arrivée une étudiante polonaise en études théâtrales avait réalisé en cette école son terrain pour un mémoire de master.

18 En anglais : Balakrishnan (1995a), Sreedharan Nair (2007). En malayalam : Balakrishnan (1995b), Sreedharan Nair (1963), Vasudeva (2011), Vijayakumar (2000).

19 C'est aussi l'âge indiqué par les témoignages de voyageurs européens des $16^{\mathrm{e}}$ et $17^{\mathrm{e}}$ siècles sur l'entraînement des guerriers au Malabar. Voir encore Duarte Barbosa (1946 : 139-140). 
été remise en question par certains maîtres reconnus ${ }^{20}$, douze ans sont généralement jugés nécessaires pour acquérir non seulement la maîtrise technique des exercices, mais aussi leur compréhension profonde et certaines capacités perceptives spécifiques. Toutes ces qualités pourront éventuellement conduire le pratiquant, après avoir atteint la quarantaine, à devenir maître (gurukkal).

Seule une minorité des pratiquants atteint le dernier niveau, puisque la plupart abandonne avant. Enfin, une partie des apprentis continuera à pratiquer sans parvenir pour autant à recevoir la transmission des connaissances du maître dans leur intégralité. Le discours ambiant veut que les techniques les plus avancées, étant donné leur dangerosité potentielle, soient enseignées uniquement aux disciples les plus méritants (nous reviendrons plus tard sur ce qui définit le mérite).

Parmi les connaissances les moins divulguées figurent, en plus des recettes des préparations thérapeutiques, les connaissances relatives aux points vulnérables du corps, les marmmaṇ, qui constituent l'un des éléments de base de la tradition martiale de l'Inde du sud (Roşu, 1981 ; Sieler, 2015 ; Zarrilli, 1992a, 1992b). Les marmmam sont définis comme des points du corps où le passage du souffle vital (prāṇa) est plus intense et qui, atteints d'une façon spécifique, peuvent entraîner des blessures graves, voire la mort. Ces mêmes points peuvent toutefois servir également à des fins thérapeutiques. Une grande partie des mouvements composant les séquences d'entraînement au kalarippayarru aurait pour objet l'attaque ou la protection de ces points, mais ce n'est qu'aux dernières étapes de l'apprentissage que ces applications sont éventuellement enseignées, en même temps que les manœuvres à effectuer pour remédier à leur atteinte. Par ailleurs, ces points ne sont ni visibles, ni repérables sans un apprentissage spécifique pour les détecter et les traiter. Le terme sanscrit marman, dérivé de la racine $m r$ - (« mourir »), indique dans la médecine ayurvédique les points du corps qui ne doivent pas être percés, mais en malayalam (marmmam), il en est venu à indiquer ce qui est secret et caché, ou bien l'habileté de qui connaît les arcanes d'un métier ou d'un art (Zarrilli, 1992a ; Sieler, 2015 : 39).

La notion de souffle vital sous-jacente à la connaissance des marmmam se rattache aussi, pour certains, à une série de notions plus ou moins articulées sur la circulation du souffle vital dans le corps. Celles-ci composent la géographie du corps communément dit « subtil » dans les langues européennes ${ }^{21}$, constituée de canaux invisibles ( $\left.n \bar{a} d \bar{l}\right)$ qui véhiculent le souffle vital, et pour certains de centres (cakra ou ādhāra) situés le long de l'axe vertébral, image du corps proche du yoga tantrique à laquelle certains maitres

20 Le célèbre Chirakkal T. Sreedharan Nair ( $c f$. note 7), qui avait commencé son apprentissage à l'âge de 21 ans après avoir pratiqué l'haltérophilie et la lutte, soutenait qu'un adulte en bonne condition physique pouvait maîtriser les fondements du kalarippayarru en quelques mois de pratique régulière (Sreedharan Nair, 1963 : ix).

21 André Padoux (2002 : 171-173; 2010 : 126) a remarqué que l'expression corps subtil correspond littéralement à l'idée du sūkșma śārīra, l'ensemble des éléments de la personne qui transmigre d'un corps à un autre. Toutefois elle est utilisée dans plusieurs langues européennes pour se référer à ce qu'on pourrait plutôt appeler corps imaginal, support de méditation pour les adeptes du hathayoga. Le maître en question associe les expressions subtle body (corps subtil) et sūkșma śārīra, entendant dans les deux cas ce que Padoux appelle corps imaginal. 
adhèrent mais dont ils n'évoquent pas volontiers les détails ${ }^{22}$. Il s'agit en fait de modalités d'usage de l'énergie et de perceptions qu'à leur avis il faudrait expérimenter à travers une pratique assidue plutôt que de concepts à saisir intellectuellement. La monographie de Zarrilli (1998) leur consacre toutefois un chapitre rapportant les remarques de plusieurs maîtres sur ce sujet. Ce livre est vendu dans la plupart des librairies au Kerala et il est bien connu des pratiquants. Certains reprochent cependant à cet auteur d'avoir trop écrit sur ce qu'ils appellent « la spiritualité » et d'avoir consacré une grande place à des notions qu'ils perçoivent comme peu présentes dans leur pratique ou qui, en tout cas, ne devraient pas être exposées à des débutants, entre autre pour ne pas affecter la progression de leur apprentissage.

Les disciples sont admis graduellement dans des espaces de connaissance qui leur sont celés tant que le maître ne décide pas de leur révéler tel ou tel aspect technique. Dans quelques cas il s'agit d'une exclusion matérielle, les disciples les plus avancés recevant des enseignements en des moments ou en des lieux où les autres ne peuvent pas voir ce qui se passe. Dans d'autres cas, la connaissance ou la méconnaissance de certains détails techniques des exercices pratiqués suffisent à constituer une cloison invisible qui sépare deux niveaux de compétence. Il existe donc plusieurs coulisses, matérielles et métaphoriques, auxquelles les différents apprentis peuvent avoir accès en des moments différents ${ }^{23}$.

« Enseigner le kalarippayarru à une personne indigne, me disait un jour le fils du maître, c'est comme tuer une femme enceinte : c'est un crime double ». Le mérite ne s'apprécie pas simplement sur la base d'une plus ou moins grande habileté technique, mais aussi sur la présence de certains traits de personnalité et de qualités morales comme la constance, la motivation, la fidélité, la loyauté, l'humilité et le respect du maître et des condisciples. Il est bien connu que dans le monde indien la relation entre maître et disciple fait l'objet d'un fort investissement affectif et symbolique. Dans l'idéal, un bon disciple se confie totalement à son maître, est fidèle et obéissant et accepte humblement toutes ses décisions, en particulier celles qui concernent la progression de son apprentissage. Un autre critère très important est de ne pas aimer la violence et de ne pas vouloir apprendre les techniques martiales pour s'en servir en dehors du kalari. Cependant, cet enseignement privilégié s'adresse souvent de préférence au fils ou au neveu (fils de la sœur) du maître ${ }^{24}$. Par ailleurs, comme le remarquent tant

22 Ils peuvent d'ailleurs, comme le note Phillip Zarrilli (2005 : 20), suivre différents « paradigmes de pratique et d'enseignement » (« paradigms of teaching and practice »), qui ne se basent pas sur ces conceptions. Sur un fond commun de physiologie humorale, les positions concernant la pratique correcte et les notions sous-jacentes sont très variables.

23 Dans Les cadres de l'expérience, Goffman mentionne « les barrières à communication, les cloisons, les distances ou la ségrégation des publics » (1991:171) comme des éléments qui contribuent à la réalisation des fabrications. Il définit celles-ci comme « des efforts délibérés, individuels ou collectifs, destinés à désorienter l'activité d'un individu ou d'un ensemble d'individus et qui vont jusqu'à fausser leurs convictions sur le cours des choses » (Goffman, 1991 : 93). Les fabrications et le comportement régional présentent alors des éléments en commun, dont le plus important est l'effort conscient de cacher à un public une part de la réalité. Toutefois, dans la mesure où le but des maîtres d'arts martiaux est principalement de réguler la transmission du savoir et non pas tant de fourvoyer les disciples, les métaphores régionales me semblent plus pertinentes en ce contexte. 
Phillip Zarrilli (1992a) que Roman Sieler (2015), et comme j'ai pu l'entendre dire par plusieurs pratiquants, il existe une idée reçue selon laquelle certains gurukkal ne sont pas disposés à transmettre leurs connaissances les plus précieuses même à leurs disciples les plus fidèles, et considèrent qu'ils ne devraient les révéler que sur leur lit de mort. Cela a favorisé l'opinion commune que, puisque plusieurs gurukkal seraient décédés sans avoir révélé leurs secrets, la connaissance globale de la discipline s'amoindrit progressivement, une opinion qui renforce l'idée de l'ancienneté et de l'ampleur du savoir transmis dans les kalari avant la domination britannique. Les savoirs spécialisés constituent le capital symbolique, social et potentiellement économique de ceux qui en sont les détenteurs ${ }^{25}$. Il existe un équilibre, délicat à trouver, entre transmission et rétention de l'information. Pour être connus et exercer leur métier, les maîtres doivent forcément divulguer une partie de leurs connaissances ; pour avoir des disciples et/ou des patients, ils doivent mettre en avant leur compétence technique et ils ne peuvent pas gérer une école sans la transmettre. En même temps, ils doivent garder précieusement leur capital, et doivent aussi faire attention à respecter la progression des disciples s'ils souhaitent que leur apprentissage soit solide. Cette tension donne lieu aussi à des fantasmes de la part des disciples et parfois à des accusations réciproques de la part des maîtres. D'après certains d'entre eux, tel ou tel se réfugierait derrière le secret pour cacher son incompétence.

Roman Sieler, auteur d'une étude (2015) sur les points vitaux dans le varma kalai, une tradition martiale et thérapeutique proche du kalarippayarru pratiquée principalement au Tamil $\mathrm{Nadu}^{26}$, Etat voisin du Kerala, discute longuement la dialectique entre secret et révélation concernant les marmmam/varmam (ce dernier est le terme tamoul). Les maîtres de varma kalai (les $\bar{a} c \bar{a} \underline{n}$ ) sont renommés pour leur capacité de soigner en agissant manuellement sur les points vitaux. Quand ils opèrent dans leur cabinet ils se cachent derrière un rideau pour que personne ne puisse observer la technique utilisée, créant ainsi une coulisse. Lorsqu'une urgence les amène à agir dans la rue, ils couvrent le patient avec un drap, en recréant ainsi une région invisible au public. La norme explicite est qu'un vrai maître ne révèle pas ses techniques. Un vrai $\bar{a} c \bar{a} \underline{n}$ maintient le secret sur ses connaissances et par conséquent, celui qui parle ou montre ses techniques n'est pas un vrai $\bar{a} c \bar{a} \underline{n}$. Sieler (2015: 182-183) rapporte même le cas d'un maître qui a recours à un expédient pour éloigner son assistant lorsqu'il doit appliquer une technique qu'il ne souhaite pas lui montrer. Sieler montre comment l'aura de secret qui entoure les varmam a non seulement pour effet d'établir l'autorité des pratiquants, mais aussi de situer l'art des $\bar{a} c \bar{a} \underline{n}$ par rapport à l'hégémonie de la biomédecine en Inde. Invisibles à l'œil humain et aux appareils diagnostiques biomédicaux, les varmam sont au-delà des investigations mises en œuvre dans la tradition médicale dominante. Toutefois, ces mêmes maîtres qui insistent sur le secret se livrent de temps en temps à des démonstrations publiques de leurs capacités. Par ailleurs, bien que la démonstration soit ouvertement stigmatisée, plusieurs $\bar{a} c \bar{a} \underline{\underline{n}}$ ont montré au chercheur quelques

25 Marie-Claude Mahias écrit à ce propos : « De manière générale, la transmission et la reconnaissance des savoirs techniques, et plus encore leur appropriation et leur transmission, sont toujours au cœur des rapports sociaux. Elles constituent des instruments de pouvoir et des enjeux sociopolitiques, et font toujours l'objet d'un contrôle social. » (Mahias, $2011: 16$ )

26 On trouve aussi des pratiquants au Kerala, spécialement dans le sud de l’Etat. 
aspects de leur pratique sur les varmam, en lui faisant promettre de ne pas divulguer l'enseignement reçu. Bien que Sieler affirme respecter la norme de la discrétion qui entoure la pratique, un maître lui aurait promis de tout lui montrer (Sieler, 2015 : 194). Par ailleurs, tous lui ont dit que toute technique que d'autres peuvent lui avoir montrée ne peut qu'être fausse, puisqu'il n'y a que les charlatans qui donnent des démonstrations ou qui révèlent les détails de leur travail. Le dévoilement de ses compétences prive le pratiquant de sa protection et en fait facilement la cible des évaluations et critiques de la part de ses compères ${ }^{27}$. L'étude de Sieler met ainsi en évidence un paradoxe qui n'est pas spécifique à ce contexte : une information ou une connaissance, acquiert d'autant plus d'importance qu'elle est rare et peu accessible. Toutefois, pour qu'elle confère du prestige à ses détenteurs, il faut que l'existence de l'information cachée soit connue. C'est ce que Sieler appelle « la publicité du secret ${ }^{28}$ », qui fait que « le secret n'existe que dans une dialectique entre dissimulation et révélation ${ }^{29} »$. Ce qui importe alors, n'est plus tant de connaître le contenu caché - si vraiment il y en $\mathrm{a}^{30}$ - mais ce sont les stratégies adoptées par les acteurs pour révéler ce qui doit être maintenu secret tout en conservant son prestige. Si le paradoxe du secret avec les questions qu'il pose aux chercheurs a déjà été reconnu et analysé ${ }^{31}$, Sieler estime que : "il faudra consacrer plus d'attention à l'avenir aux notions de dissimulation, de révélation et ou de secret en tant qu'éléments discursifs et performatifs ${ }^{32} »$. Il me semble que ce problème pourrait bien être abordé à l'aide des outils élaborés par Goffman, spécialement dans la mesure où les représentations analysées par cet auteur ne sont pas forcément des fictions. Une mise en scène est en même temps la mise en acte d'une réalité sociale. La distance, observe déjà Goffman (1973 : 70-71), est un élément essentiel de la façade qui constitue la représentation de l'autorité et du prestige. Nous avons vu que les maîtres de varma kalai peuvent parfois se cacher dans une coulisse matérielle. Toutefois, la plupart du temps leur coulisse est une région métaphorique, comme elle l'est pour les maîtres de kalarippayarru. C'est ce qu'avait expérimenté Berreman, à travers la manière dont les villageois auprès de qui il menait son enquête choisissaient de retenir ou de révéler certaines informations. En dosant et maîtrisant les impressions destinées à l'ethnographe, les habitants du village himalayen visité par Berreman cherchaient aussi à sauvegarder les relations qu'ils entretenaient avec les autres villageois. De la même manière, la relation qu'un maître entretient avec ses disciples peut avoir un impact sur ses relations avec les autres maîtres et sur l'opinion que le public se fera de lui.

27 Ce qui peut fournir un élément de compréhension supplémentaire des critiques émises à l'encontre du livre de Phillip Zarrilli.

28 «Advertisement of secrecy » (Sieler, $2015: 179)$.

29 « Secrecy thus only exists in a dialectic between concealment and revelation » (Sieler, 2015 : 193).

30 Goffman aborde brièvement cette question dans le premier tome de La mise en scène de la vie quotidienne, dans un paragraphe consacré à la mystification (1973:68-71).

31 Sieler fait référence à plusieurs travaux en sciences humaines et sociales qui abordent cette question sous différents angles disciplinaires.

32 « The predicaments of concealment and revelation or of secrecy as discursive and performative elements still require further attention » (Sieler, $2015: 206)$. 


\section{Malentendus et déplacement des coulisses}

Au début de l'enquête, ma connaissance de la transmission du kalarippayarru correspondait au modèle idéal mentionné dans le paragraphe précédent. D'ailleurs, avant de me rendre sur place, la personne qui m'avait mise en contact avec l'école, une enseignante de yoga expérimentée, m'avait prévenue de la réticence des maîtres, de leur méfiance vis-à-vis des profanes et de l'importance de la fidélité au maître choisi ${ }^{33}$. L'idée était que souvent les étrangers souhaitent s'approprier trop vite des savoirs locaux pour en tirer des profits faciles une fois revenus dans leur pays. Il m'apparaissait alors indispensable de m'exercer comme tout autre apprenti, avec la conviction que les gurukka! n'auraient partagé leurs connaissances qu'avec des disciples qu'ils estimaient sérieux et loyaux. La fidélité à la pratique d'un seul maître participait de ce sérieux. Mon objectif n'était d'ailleurs ni de devenir une praticienne experte, ni d'arriver à saisir en profondeur toutes les techniques dans le cadre de mon doctorat. Je m'intéressais aux conceptions du corps dans leur ensemble et je souhaitais réussir à en connaître quelque chose en participant à la vie quotidienne de l'école plutôt qu'en interrogeant directement les pratiquants ${ }^{34}$. Pendant les cinq premiers mois j'ai donc suivi les entraînements dans la mesure du possible, sans m'attendre à avancer beaucoup dans l'apprentissage et en laissant aux enseignants le soin de décider de ma progression. Les discours tenus par le maître et par ses enfants confirmaient l'importance de protéger le savoir martial en ne le divulguant qu'avec la plus grande prudence, ce qui m'encourageait à maintenir la ligne de discrétion que j'avais choisie. Toutefois, plusieurs faits sont bientôt venus contredire ces discours. Tout d'abord, la progression de l'apprentissage qui m'était proposée était beaucoup plus rapide que je ne m'y attendais. La situation s'est compliquée quand, après moins de trois mois, le maître a commencé à évoquer l'idée que je puisse devenir enseignante de kalarippayarr $u^{35}$. Connaissant les discours ambiants sur l'importance de pratiquer de nombreuses années avant de passer à l'enseignement et les ayant également entendus dans l'entourage du maître, j'étais à la fois flattée et perplexe. Cinq ou six mois après mon arrivée, le nombre assez conséquent d'enseignants certifiés (au moins une douzaine) que l'école avait formés ou était en train de former, parfois en quelques mois, a aussi contribué à remettre en question la rhétorique sur le temps de l'apprentissage, à laquelle j'avais cru sans réserve. Me préparer à enseigner aurait signifié m'investir totalement dans la pratique et renoncer à consacrer du temps et de l'énergie à l'étude du malayalam, ainsi qu'à l'exploration de tout ce qui entoure l'école et à la découverte d'écoles différentes. En empruntant ce chemin, j'aurais dû renoncer à mon entreprise de connaissance telle que je l'entendais à ce moment-là et envisager une entreprise différente. Sans tout de suite opposer au

33 Dans les faits, plusieurs pratiquants se rapprochent de divers maîtres successivement, en cumulant les techniques transmises par les uns et par les autres.

34 Je prévoyais d'effectuer des visites dans d'autres écoles pour mettre en perspective ce que j'apprenais tout en maintenant ma fidélité pratique au maître de l'école où j'étais installée. Je comptais alors m'en tenir à l'enquête orale.

35 Mon niveau à ce moment-là se situait entre débutant et intermédiaire. 
maître un refus explicite, j'ai donc renoncé à cette proposition ${ }^{36}$. Je croyais aussi montrer de cette manière la sincérité de ma motivation à connaître le kalarippayarr tout en respectant les règles $d u j^{3} u^{37}$, même si cela me coûterait plusieurs années. A partir des discours courants sur la durée de l'apprentissage et sur la prudence requise dans la transmission des savoirs, je m'étais d'ailleurs construit une idée selon laquelle le maître se voyait obligé d'autoriser rapidement l'enseignement pour répondre aux demandes de sa clientèle, mais qu'il le faisait à contrecœur. Je tenais alors à me montrer respectueuse de la réticence que je lui avais attribuée sur la base de mes connaissances préalables. Or, en me conformant à une norme explicite, j'en transgressais une autre, plus importante mais implicite. La déception du gurukkal, manifestée par la réitération de sa proposition et par la perte d'intérêt envers la progression de ma pratique, m'a fait comprendre que, contrairement à ce que la rhétorique traditionaliste me faisait croire, les étrangers motivés à enseigner dans leur pays sont des ambassadeurs importants qui vont constituer la façade du kalarippayarru et de l'école au niveau international même si leur apprentissage n'est pas encore complet. Ils sont autorisés à transmettre des enseignements de base en tant qu'enseignants, et non comme maîtres (gurukkal), et continuent ensuite à se perfectionner en revenant à l'école pour des séjours réguliers. D'ailleurs, dans beaucoup de kalari au Kerala les disciples les plus avancés collaborent à l'enseignement destiné aux plus jeunes.

Trois autres raisons pourraient expliquer pourquoi ce maître ne se conforme pas à la temporalité idéale de l'apprentissage. Tout d'abord, sa référence à l'enseignement de Sreedharan Nair, l'une des principales figures fondatrices du kalarippayarru actuel, lequel avait rompu avec l'idée qu'un très long apprentissage était nécessaire pour tout novice, estimant qu'une personne adulte en bonne condition physique pouvait avancer très rapidement (Sreedharan Nair, 1963 : ix). Le maître ne se réfère pas explicitement à cet aspect de l'enseignement de Sreedharan Nair bien qu'il l'évoque de temps en temps, mais il le suit dans les faits, et ceci d'autant plus que les disciples étrangers ont souvent déjà acquis une formation dans d'autres pratiques corporelles, que ce soit un art martial, la danse ou le yoga. Ensuite, le fait que toutes les précautions à prendre avant de transmettre des enseignements aux disciples indiens ne s'appliquent pas forcément aux étrangers, lesquels peuvent difficilement entrer en compétition avec le maître sur son propre terrain (Luijendijk, 2008 : 73). Cela pourrait expliquer que ce maître se montre plus ouvert envers les disciples étrangers qu'envers les Indiens dans la transmission non seulement des techniques mais aussi des élaborations conceptuelles qui les accompagnent. Les disciples destinés à repartir dans leurs pays d'origine échappent d'une certaine manière au contrôle social qui gouverne la transmission de la connaissance au Kerala. Il semblerait que pour eux l'entrée dans les coulisses ${ }^{38}$ se fasse

36 J'ai été aidée en ce choix par une blessure physique qui m'a à la fois obligée à diminuer mes entraînements et permis de consacrer du temps à autre chose.

37 Avant de partir sur le terrain je m'étais appuyée sur l'ouvrage de Loïc Wacquant (2002), où l'auteur étudie la constitution de l'habitus des boxeurs d'un club de Chicago en se soumettant entièrement au parcours d'apprentissage.

38 J'utilise ici le pluriel parce que, comme j'espère l'avoir montré, il peut exister plusieurs coulisses dans lesquelles on est admis ou pas selon son avancement et selon le type de relation entretenue avec le maître. 
plus vite que pour les disciples locaux ${ }^{39}$, même si le fait de pratiquer des techniques avancées ne signifie pas forcément que l'on soit un pratiquant avancé, car tous les niveaux de compréhension de la pratique pourraient ne pas avoir été atteints. Enfin, la circulation de plus en plus rapide de l'information à travers la presse et l'internet permet au maître d'affirmer que les disciples étrangers sont au départ plus informés que les Kéralais.

J'ajouterai que le discours du maître à mon égard différait parfois de celui qu'il tenait à ses autres disciples. Comme je l'ai évoqué plus haut, il avait l'habitude de satisfaire partiellement leur désir d'apprendre lors du moment de convivialité du déjeuner, ou bien pendant les trajets entre les logements et le kalari. Ses réponses obéissaient à la logique exposée par Roman Sieler (2015 : ch. 5) : laisser entrevoir qu'il y a beaucoup à découvrir et motiver à l'apprentissage, sans toutefois donner accès à une compréhension complète ; le moment pour celle-ci, déclarait-il, n'étant pas encore venu. Or, en l'absence des autres disciples, il m'encourageait à lui poser des questions, insistant, comme ce fut le cas pour Roman Sieler, sur le fait qu'il me dirait tout et qu'il n'y aurait pas de secrets. Ces invitations se sont multipliées quand il a compris que, contrairement à ses attentes et suppositions, je n'étais pas là pour faire de l'enseignement du kalarippayarru mon activité professionnelle mais que, tout en souhaitant participer à la pratique, mon but principal était une production intellectuelle, comme je l'avais annoncé dès le départ. Cela finissait par m'amener à me soustraire en partie à la routine de l'école pour me consacrer à l'apprentissage du malayalam, à la recherche bibliographique ou à la traduction de textes. Une autre supposition a alors remplacé la précédente : il s'attendait à ce que je lui demande explicitement ce que je souhaitais connaître, comme l'avait fait l'autre chercheur qui était venu le trouver. Pour ma part, sa volonté de satisfaire mes attentes m'a surprise, puisque je partais du principe que c'était aux disciples de se conformer aux attentes du maître et non l'inverse. Le maître comme moi avons dû renoncer à nos suppositions réciproques : le maître, à l'idée que les étrangers venaient soit pour faire du tourisme, soit pour se professionnaliser ; moi, à l'idée que le gurukkal serait peu disposé à transmettre son enseignement, spécialement concernant les conceptions du corps.

Poser des questions dans des moments et des lieux explicitement consacrés à ce type d'échanges était exactement ce que je cherchais à éviter, jusqu'à ce que je comprenne, vu le contexte, la nécessité de m'adapter et de considérer cette modalité de communication comme une partie de ce que j'apprenais sur ce terrain. J'ai toutefois décidé de ne demander des précisions qu'à partir des exercices que je connaissais. J'ai dès lors été plus souvent admise dans cette coulisse apparente (nous verrons pourquoi) qu'est la maison du gurukkal. Cela m'a aussi permis d'observer comment le maître pouvait déplacer le seuil entre la région antérieure et la coulisse, selon ses interactions avec les différents disciples. Voyons un exemple. Une après-midi, seuls un disciple récemment arrivé - mais très prometteur - et moi-même étions restés dans la salle à

39 Bien que ce maître ait été reconnu localement pour la qualité de sa pratique et de ses connaissances, ce type d'approche n'est pas sans attirer des jugements négatifs de la part d'autres pratiquants au Kerala. Actuellement, il est l'objet de critiques tant pour le contenu du livre de Luijendijk (2008), totalement inspiré par ses enseignements, que pour le tournant entrepreneurial pris par son école. Comme nous l'avons vu, le fait de révéler ses connaissances et ses réflexions ne revient pas seulement à céder une part de son capital, mais prive aussi le maître d'une forme de protection à l'encontre de ses pairs. 
manger. Le maître demanda alors à ce disciple de lui prendre le pouls. Puis, du pouce et de l'index, il pressa un point derrière son oreille et lui demanda de lui reprendre le pouls, lequel avait ralenti entre temps. Face à ses demandes d'explications, le maître enjoignit à son disciple de poursuivre son apprentissage : le moment opportun viendrait pour apprendre cette technique. Ce disciple et moi étions à ce moment-là dans une coulisse par rapport aux autres disciples, mais nous nous trouvions encore une fois dans une région antérieure par rapport au maître. Ce qu'il nous offrait, c'était une représentation.

Chez le gurukkal j'ai pourtant pu recevoir un enseignement sur ses élaborations théoriques concernant la circulation du souffle vital, la physiologie humorale, les effets des exercices sur le corps, l'esprit et les émotions des pratiquants, des notions auxquelles j'avais cru ne pouvoir accéder qu'à travers mon avancement dans la pratique. Un jour, en m'entretenant avec un pratiquant plus avancé que moi, il m'est arrivé de mentionner quelques notions dont je pensais qu'il avait connaissance, et j'ai découvert que ces informations ne lui avaient pas été transmises. J'aurais pu me croire dans une coulisse mais je n'étais en fait que dans une autre région antérieure et je savais que d'autres recevaient d'autres fractions de l'enseignement ${ }^{40}$. Quelques jours avant mon départ du Kerala, j'ai à mon tour été exclue d'une coulisse, en devant m'éloigner du lieu d'entraînement avant la fin de la séance. La représentation, en anglais, voulait que pour des raisons organisationnelles liées à la disponibilité des voitures, je rentre avant les autres. Mais en fait, en coulisse, la discussion en malayalam entre les assistants du maître dont j'arrivais à saisir quelques bribes, m'a permis de comprendre qu'il n'était pas souhaitable que j'assiste à l'entraînement d'un disciple qui, à ce momentlà, entreprenait l'étude des marmmaṇ. J'ai parlé d'une cloison invisible formée non seulement par la langue, mais aussi par l'organisation de la journée dans l'école. Au moment où j'ai cherché à traverser cette cloison en décidant différemment de mon emploi du temps, j'ai obtenu un aperçu d'un espace qui m'était caché auparavant, mais j'ai été exclue d'autres.

En guise de conclusion, on peut arguer que la chercheuse étrangère qui enquête sur le kalarippayarru se trouve impliquée dans deux dynamiques déjà complexes en ellesmêmes. D'un côté, la relation entre les visiteurs venant de l'étranger et les habitants, pour qui les premiers représentent une chance à saisir mais également un risque. De l'autre côté, la relation entre le maître d'armes et ses disciples, une relation fortement investie socialement et culturellement, où la gestion de l'information de la part du maître répond à différentes exigences pédagogiques, économiques et stratégiques. Les métaphores de Goffman m'ont paru fournir un outil pertinent, spécialement à la lumière de la reprise qu'en a faite Berreman, pour analyser la manière dont les gurukkal négocient les passages d'information dans leurs relations tant avec les disciples indiens et étrangers, qu'avec leurs pairs et avec la société environnante. Cette même perspective m'a également permis d'analyser ma propre interaction avec le maître. Il attendait de moi qu'en tant que praticienne, je me prépare à faire connaître et à transmettre la discipline dans mon pays mais aussi que je contribue à sa dissémination à travers un livre en tant que chercheuse. De cette manière, j'aurais contribué au rayonnement de

40 Le recoupement des ces informations avec les conversations que j'ai eues avec d'autres maîtres au Kerala et avec des pratiquants de kalarippayarru kéralais et français vivant en France m'ont permis de construire un cadre dans lequel situer et interpréter les réponses du maître. 
son école (et à l'amplification de sa représentation ?) et de la discipline à laquelle le maître a consacré tant d'efforts, plus que je ne l'ai fait en prenant mes distances par rapport au rôle qui m'était attribué. C'est pourtant cette rupture qui m'a permis de saisir des enjeux de la transmission que j'aurais probablement laissés passer si je n'avais pas perturbé ce qui, dans la perspective de Goffman, pourrait être appelée la représentation que l'école et moi, nous sommes réciproquement offerte.

\section{Références citées}

Ashley, Wayne, 1993. Recodings : Ritual, theatre, and political display in Kerala State, South India. Thèse de doctorat, Department of Performance Studies, New York University.

Balakrishnan, P., 1995a [1994]. Kalarippayarrụ. Keralattile pracina ayōdhana mura. Trivandrum : C.V.N. Kalari.

- 1995b. Kalarippayattu. The ancient martial art of Kerala. Trivandrum : C.V.N. Kalari.

Barbosa, Duarte, 1946 [1516]. Livro em que dá relação do que viu e ouviu no Oriente. Lisboa : Agência Geral das Colónias. <http://purl.pt/435>.

BAyly, Susan, 1989. Saints, goddesses and kings. Muslim and Christians in South Indian society, 1700-1900. Cambridge : Cambridge University Press.

Berreman, Gerald Duane, 1962. Behind many masks, ethnography and impression management in a Himalayan village. Lexington : Society for Applied Anthropology.

-, 1972. « Behind many masks, ethnography and impression management in a Himalayan village », in Gerald D. Berreman, Hindus of the Himalayas. Ethnography and change. Berkeley/Los Angeles : University of California Press, pp. xvii-lvii.

FAWCETT, Fred, 1915 (1901). Nayars of Malabar. Madras : Government Press.

Goffman, Erving, 1959. The Presentation of Self in Everyday Life. New York : Doubleday Anchor Books.

-, 1973. La mise en scène de la vie quotidienne. Vol. I. La présentation de soi. Traduit de l'anglais par Alain Kihm. Paris : Editions de Minuit.

-, 1991. Les cadres de l'expérience. Traduit de l'anglais par Isaac Joseph avec Michel Dartevelle et Pascale Joseph. Paris : Editions de Minuit.

Guillebaud, Christine, 2007. « Orchestrer la culture : musique et politique au Kerala (Inde du Sud) ». Actes du $3^{e}$ Congrès du Réseau-Asie, Paris, 26-28 septembre 2007.

-, 2011. "Music and politics in Kerala : Hindu nationalists versus marxists », in Daniela Berti, Nicolas Jaoul et Pralay Kanungo (eds), Cultural entrenchment of Hindutva. Local mediations and forms of knowledge. New Delhi : Routledge, pp. 29-63.

Kurup, K. K. N. et K. Vijayakumar, 1997. Kalarippayatt: The Martial Art of Kerala. Thiruvananthapuram : Department of Public Relations, Government of Kerala.

Le Breton, David, 2008. L'interactionnisme symbolique. Paris : PUF.

LuIJENDIJK, Dijk H., 2005. Kalarippayatt : India's ancient martial art. Boulder : Paladin Press.

-, 2008. Kalarippayatt : The structure and essence of an Indian martial art. Den Haag : Cip-Gegevens Koninklijke Biliotheek.

Mahias, Marie-Claude (éd.), 2011. Construire les savoirs dans l'action. Apprentissages et enjeux sociaux en Asie du sud. Paris : Editions de l'EHESS.

Mathew, Kuzhippalli S., 1979. Society in Medieval Malabar : A study based on Vadakkan Pattukal. Kottayam : Jaffe Books. 
Olivier de SARdAn, Jean-Pierre, 1995. « La politique du terrain », Enquête, 1, mise en ligne le 10 juillet 2013, consulté le 25 octobre 2015. <http://enquete.revues.org/263>.

-, 2000. « Le 'Je' méthodologique. Implication et explicitation dans l'enquête de terrain », Revue française de sociologie, 41 (3), pp. 417-445.

Padoux, André, 2002. "Corps et cosmos. L’image du corps du yogin tantrique », in Véronique Bouillier et Gilles Tarabout (éds), Images du corps dans le monde hindou, pp. 163-187. Paris : CNRS Editions.

-, 2010. Comprendre le tantrisme. Les sources hindoues. Paris : Albin Michel.

Roșu, Arion, 1981. « Les marman et les arts martiaux indiens », Journal Asiatique, CCLXIX, pp. 417-451.

SIELER, Roman, 2015. Lethal spots, vital secrets. Medicine and martial arts in South India. New York : Oxford University Press.

Sreedharan Nair, Chirakkal T., 1963. Kalarippayarru. Cannanore : Cannanore Printing.

-, 2007. Kalarippayattu. The complete guide to Kerala's ancient martial art. Chennai : Westland Books.

TARABout, Gilles, 1986. Sacrifier et donner à voir en pays Malabar. Les fêtes de temple au Kérala (Inde du Sud) : étude anthropologique. Paris : Ecole Française d'Extrême-Orient.

-, 1991. « Au 'Royaume' des brahmanes, les guerriers sont rois. Souveraineté, pouvoir et statut au Kérala », in Jacques Pouchepadass et Henri Stern (éds), De la royauté à l'état. Anthropologie et histoire du politique dans le monde indien, pp. 75-121. Paris : Editions de 1'EHESS.

-, 1997. «La mise en culture des rites », Cultures en mouvement, 5, pp. 40-43.

-, 2003. « Passage à l'art. L'adaptation d'un culte sud-indien au patronage artistique », in Yolaine Escande et Jean-Marie Schaeffer (éds), L'Esthétique : Europe, Chine et ailleurs, pp. 37-60. Paris : You-Feng.

-, 2006. "Entre normes et pratiques. L'anthropologie esthétique en Inde comme anthropologie 'politique'», Actes du $2^{e}$ congrès du Réseau Asie, Paris, 28-30 septembre 2005.

VASUDEVA, Kaduthuruthy E.P.V., 2011 [2000]. Kalarippayarrụ. Keralattinre tanatu ayōdhanakala. Kottayam : DC Books.

VIJAYAKUMAR, K., 2000. Kalarippayarrru. Keralattinre śaktiyum saundaryum. Thiruvananthapuram : Government of Kerala, Department of Cultural Publications.

WACQuAnt, Loïc, 2002. Corps et âme. Carnets ethnographiques d'un apprenti boxeur. Marseille : Agone.

WeBer, Florence, 2009. Manuel de l'ethnographe. Paris : Presses Universitaires de France.

Welton, Martin, 2011. «From floor to stage : Kalarippayattu travels », in D. S. Farrer and John WhalenBridge (éds), Martial arts as embodied knowledge: Asian traditions in a transnational world, pp. 61184. Albany : State University of New York Press.

ZARrilli, Phillip B., 1992a. « To heal and/or to harm : The vital spots (Marmmam/Varmam) in two South Indian martial traditions. Part I : Focus on Kerala's kalarippayarru », Journal of Asian Martial Arts, 1 (1), pp. 36-67.

-, 1992b, « To heal and/or To harm : The vital spots (Marmmam/Varmam) in two south Indian martial traditions. Part II : Focus on the Tamil art, Varma Ati », Journal of Asian Martial Arts, 1 (2), pp. 1-15.

-, 1998. When the body becomes all eyes. Paradigms, discourses and practices of power in Kalarippayattu, a South Indian martial art. Delhi : Oxford University Press.

-, 2005. « 'Kalarippayattu is eighty percent mental and only the remainder is physical' : Power, agency and self in a South Asian martial art », in James H. Mill (ed.), Subaltern sports. Politics and sport in South Asia, pp. 19-45. Londres : Anthem Press.

-, 2008. Psychophysical acting. An intercultural approach after Stanislasvki. Londres : Routledge. 